\title{
Dynamics of granular segregation in quasi two-dimensional system
}

\author{
Sandip H. Gharat ${ }^{1, \star}$ \\ ${ }^{1}$ Department of Chemical Engineering, Gharda Institute of Technology Lavel, Khed, Ratnagiri 415708 India.
}

\begin{abstract}
Segregation during flow of granular materials is important from an industrial point of view. Considerable work has been done on granular segregation in heap flows by continuous pouring. We studied the flow and segregation of granular mixtures during heap formation in a quasi two-dimensional rectangular bin by intermittent pouring. The heap formed by repeatedly pouring a fixed mass of the mixture. Each feeding results in the formation of a layer of the mixture on the surface of the heap. The system is a simplified model for the feeding of raw materials to a blast furnace, which is widely used for the manufacture of iron and steel. Experiments were carried out to study the dynamics of granular materials during heap formation. The number density, area fraction and average velocity of small and big particles are plotted across the flowing depth with time. Results shows that larger particles are always on top flowing over small particles. During flow small particles easily percolate through the gaps between the large particles. A thin layer of small particles is also observed at the free surface. Here the system never reached a steady state as we are pouring the mixture intermittently and system is closed. The velocity increases initially and then decreases towards the end. The number density (i.e. area fraction) profile changes for small and big particles during flow. Image analysis is done to detect the position of each particle on the side wall. Each experiment is repeated six times to get average data.
\end{abstract}

\section{Introduction}

Granular materials segregate due to difference in their sizes [1-6]. Segregation has a significant impact in industry as well as in various natural processes [7-9]. Although granular segregation on a heap has been studied earlier, in most of the cases feeding is continuous [10-15], flows on a heap by intermittent feeding are not available. In this paper, we consider the segregation during heap formation in a quasi two dimensional rectangular bin for a mixture of different sized spherical particles having equal density by means of high speed video imaging. The objective is to study the dynamics of the segregation process.

\section{Materials used}

Stainless steel (SS316) balls of different sizes (1 and $2 \mathrm{~mm}$ ) but equal in density are used as a model of granular materials.

\section{Experiments}

Experiments were carried out in a quasi two dimensional rectangular bin as shown in Fig.1. It consists of two vertical glass plates $(52 \mathrm{~cm} \times 29 \mathrm{~cm})$, aluminum divider and an auxiliary hopper. The side walls (glass plates) are transparent to facilitate imaging. The gap between two vertical glass plates is $10 \mathrm{~mm}$ and the aluminum spacer forms a bin in which the heap is formed. An aluminum divider forms an inclined chute for feeding the mixture. The auxiliary hopper is placed on the top of the bin for supply of the feed. The position of the aluminum divider can be adjusted to control the flow rate, gap $(k)$ and the height of fall to the top of the heap $(h)$. A halogen lamp (1000 watt) is used as a light source. The setup is leveled properly to ensure that side walls are vertical and the base is horizontal.

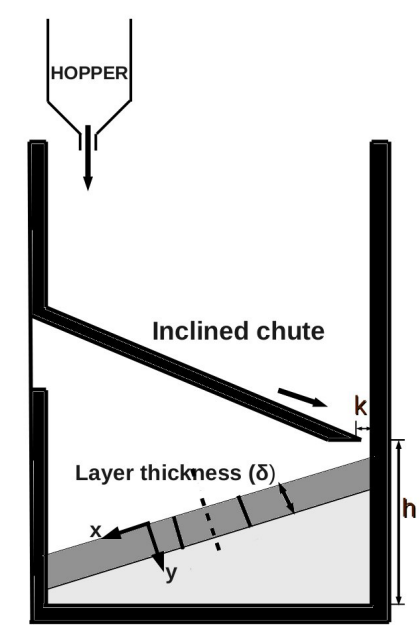

Figure 1. Schematic view of heap formation, showing feeding arrangement, feeding point, layer formed after pouring, height of feeding point, thickness of layer. The coordinate system employed in the analysis is shown.

\footnotetext{
^e-mail: sandipgharat78@gmail.com
} 


\subsection{Experimental Method}

An equal $\%$ of fixed mass of a binary mixture of different sizes is poured intermittently to form a heap. The process is repeated until the bottom part of a heap is filled. The particles were randomly mixed at the start of the experiment. A measurement was then taken for each flowing layer poured above the bottom part of a heap. A small region of flowing layer at the center of the heap (shown in dotted rectangle in figure 2a) was captured with high speed camera (Phantom 663) at 1000 frames per second. The exposure time was kept very low. In a typical capture image the particles are seen as bright white spots (a cluster of white pixels) due to reflection of light. The particles were detected using computer codes. The position of the particle was taken as mean position of the pixels present in that cluster. Small and big particles were identified based on the radius of gyration $\left(R_{g}\right)$. The radius of gyration $\left(R_{g}\right)$ is used to characterize the size of the cluster calculated as

$$
\bar{R}_{g}=\sqrt{\frac{\sum_{i=1}^{N}\left[\left(X_{i}-X_{0}\right)^{2}+\left(Y_{i}-Y_{0}\right)^{2}\right]}{N}}
$$

where, $X_{i}$ and $Y_{i}$ are the coordinates of each pixel in the cluster (see Figure 1 for definition of coordinate system) $X_{0}$ and $Y_{0}$ are center of mass of each cluster given by

$$
\begin{aligned}
X_{0} & =\sum_{i=1}^{N} \frac{X_{i}}{N} \\
Y_{0} & =\sum_{i=1}^{N} \frac{Y_{i}}{N}
\end{aligned}
$$

where $X_{0}, Y_{0}$ is taken to be the mean position of the particle.Figure (2c) shows the detected small and big particles for the original captured image. Position of each particle in all images were detected to get trajectories of each particle (small and big). From the trajectories, the velocity of each particle is determined. The co-ordinate axis is shown in figure (2a). For analysis we have taken a region of length $20 \mathrm{~mm}$ (10 mm downstream and upstream from the center) and depth of $15 \mathrm{~mm}$ (flowing layer thickness) to understand the flow of binary granular materials. The region of interest is divided into bin of 1 or $2 \mathrm{~mm}$ to the flow direction. The total number of images for each flowing layer are $7500( \pm 300)$. The total images counted is based on the material seen in the first image till it finishes. For analysis we have taken a set of 200 images starting from image no. 500 to 699 (where velocity of particle is sufficient) till the last set of 7000-7199 with the gap of 500 from staring image. (e.g., 500-699, 1000-1199 and so on). During flow, void space between the particles increases due to which small particles can easily percolate through a gap of larger particles. Deposition of small particle in turn increases the depth as flow progresses. Variation in the $y$-position affects the velocity profile therefore it is necessary to shift the y-position with the reference to get correct analysis of flow of particles. Hence, for each set of images the $y$-position is kept constant by shifting the co-ordinate (y) with the reference surface after each pouring i.e. passage of each flow.

\section{Image Analysis}

Image analysis is used to detect the position and size of the particles from captured images. The particles appear as tiny bright spots on a black background. A typical captured image is shown in Figure 2(b).

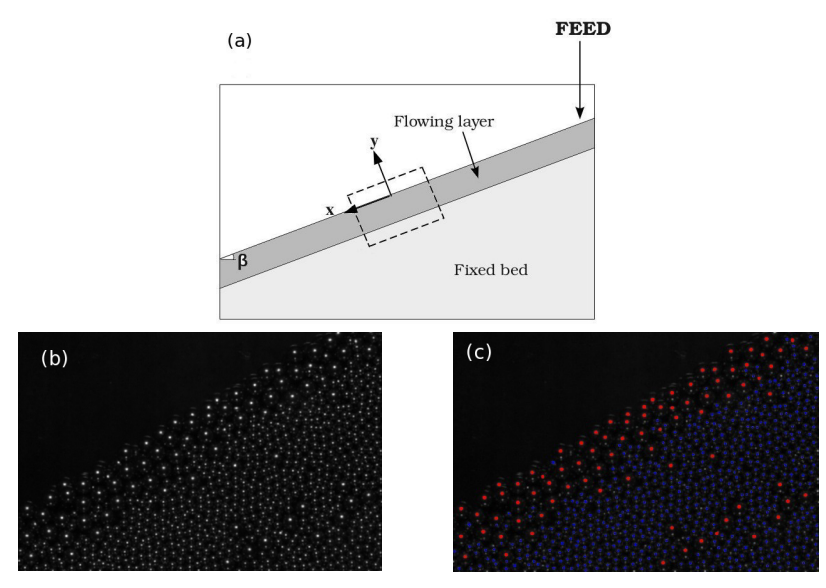

Figure 2. (a) Schematic view of the system: a flowing layer with a mixture of $50 \%$ by volume of small $(1 \mathrm{~mm})$ and $50 \%$ by volume of big $(2 \mathrm{~mm})$ spherical steel particles. The free surface makes an angle $\beta$ with the horizontal. The coordinate system used has its origin at the center as shown. The rectangular region denoted by the dashed line is captured with a high-speed camera at 1000 fps and analyzed. (b) Typical captured image: The particles are seen as bright spots for large particles and (c) Identified large (red on-line) and small (blue on-line) particles for the image shown in (b) using image analysis.

The captured gray scale images are converted into black and white images by thresholding. The threshold value is chosen such that the less illuminated particles from the inner layers are eliminated and particles from the front layer appear as a clusters of white pixels on a black background. During the thresholding process, individual pixels in an image are marked as object pixels if their value is greater than some threshold value (assuming an object to be brighter than the background) and as background pixels otherwise. The captured image after thresholding shows individual white clusters of pixels (figure 2(b)). We next scan the threshold image for a white pixel, starting from the top left corner, in both the right and the downward directions. Pixels adjacent to this pixel are scanned to identify white pixels and these are assigned to the cluster. All neighbouring pixels of pixels in the cluster are then scanned to again identify white pixels which are assigned to the cluster. The process is repeated iteratively until all pixels belonging to the cluster are identified and stored. Similarly all the white clusters in that image are identified.

The particles detected in the threshold images as white clusters are identified as large or small based on the radius of gyration of the cluster. Clusters with values of $R_{g}$ greater than or equal to 3.5 pixels are taken to be large particles and small particles otherwise. Detected particles are 
shown in figure 2(c) in red (big particles - $2 \mathrm{~mm}$ ) and blue (small particles $-1 \mathrm{~mm}$ ).

For analysis we have chosen a co-ordinate system with its origin $(x, y)$ at the top left corner of the free surface layer, such that the $+v e \mathrm{x}$-axis is opposite to the flow direction and $+v e \mathrm{y}$-axis is in the downward direction. The default and transformed co-ordinate axis is shown in figure 1. The new position of the particles are calculated using the following transformation.

$$
\begin{aligned}
& x=X \cos \theta+Y \sin \theta-X_{c} \\
& y=Y \cos \theta-X \sin \theta-Y_{c}
\end{aligned}
$$

Here $\mathrm{x}, \mathrm{y}$ and $\mathrm{X}, \mathrm{Y}$ gives the position according to the new and old coordinate system respectively. $\theta$ is the angle the free surface makes with the horizontal. $X_{c}, Y_{c}$ are coordinates of the origin.

\section{Results and Discussion}

We performed experiments by intermittently pouring mixtures of granular materials on a heap. The measurement were taken at the center of flowing layer.

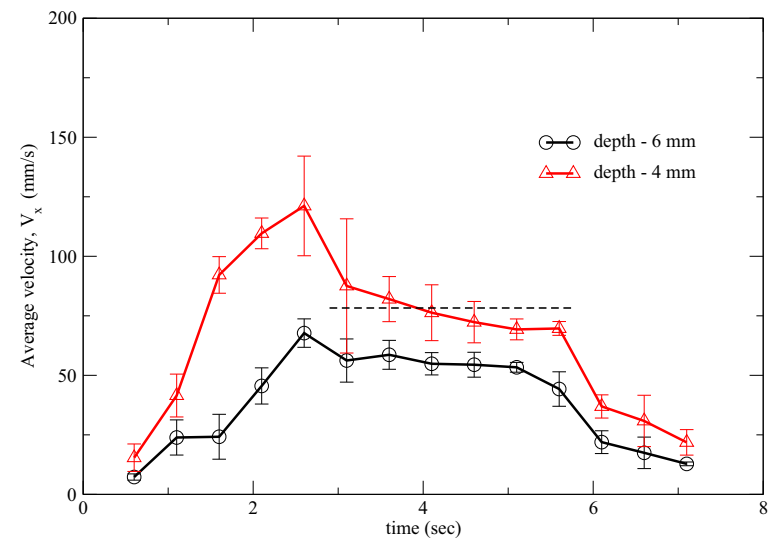

Figure 3. The mean velocity $\left(v_{x}\right)$ for small and big particles across the depth within the flowing layer versus time.

The average velocity $\left(v_{x}\right)$ are plotted for two different $y$-position with time as shown in figure 3 . The velocity $\left(v_{x}\right)$ increases initially and is then constant for a certain time and decreases in the end. The velocity decreases as the flowing depth increases, as expected. The nature of velocity profile for different $y$-position is the same. The average velocity $\left(v_{x}\right)$ and area fraction along the depth $(\mathrm{y}-$ position) are plotted for the steady state region as shown (dotted line) in figure 3 and discuss below. The average velocity profile and area fraction for an equal $\%$ of small and big particle in the mixture within flowing layer at the center is shown in figure 4 . The position having an total area upto 0.02 is shown in the graphs and total area fraction 0.5 (actual value is 0.47) is taken as the free surface (dotted line shown in fig. 4). Gray \& Kokelaar (2010) and Weiderseiner et. al. (2011) showed that the flowing layer may have a different apparent concentration that the stationary mix. The average velocity $\left(v_{x}\right)$ for large particles is less

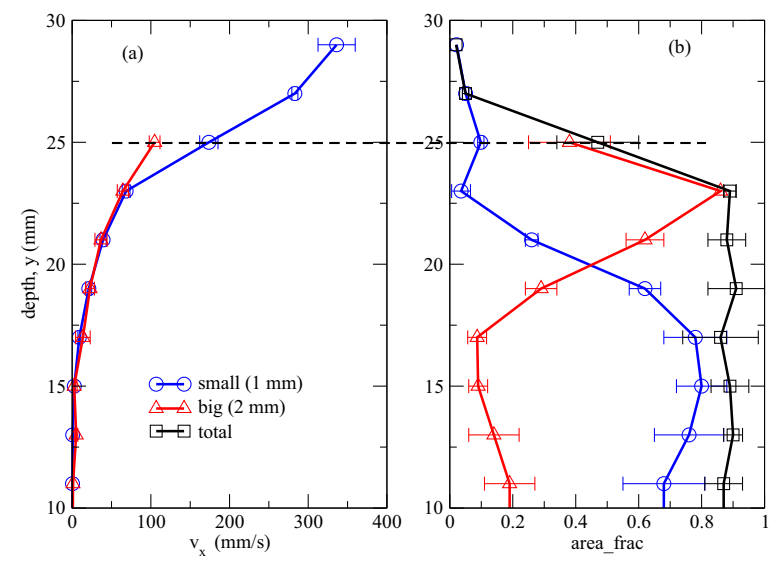

Figure 4. (a) The average velocity $\left(v_{x}\right)$ for small and big particles within flowing layer at the center. (b) The area fraction for small, big particles and total area fraction within flowing layer for mixture of $50 \%$ small and $50 \%$ big particles.

than for smaller particles near the free surface and almost the same deeper in the bed (Figure 4(a)). The velocity $\left(v_{x}\right)$ is zero near the interface and the area fraction is half of the bulk value at the free surface. The layer between the two is considered as the flowing layer.

The area fraction profile (figure $4 \mathrm{~b}$ ) shows there is partial segregation within the flowing layer as the fraction of large particles is higher near the free surface than the small particles. There is a slight deviation in total area fraction value $(0.8$ to 0.9$)$ into the bed. The area fraction for large particles is higher than for small particle within the flowing and this value decreases suddenly to zero near the free surface.

Figure 5a shows the variation in number density profile for big particles within the flowing layer across depth $(y)$ with time for a set of 50 images. We used equal \% of small and big particles as the initial mixture. The volume of pouring and the height of the divider from the bottom of the bin are $200 \mathrm{~g}$ and $22 \mathrm{~cm}$ respectively. There is a variation in number density with time. We also checked the number density profile $(n)$ for two additional sets, one is for 100 images (figure 5b) and another is for 200 images (figure $5 \mathrm{c}$ ).

Figure 5(d) shows the profiles of the number density at the three sets of images. The profiles are identical within experimental error, verifying that the all sets of images gives almost same result.

\section{Conclusions}

The segregation of equal density, different sized particles is studied to understand dynamics of segregation. The area fraction profile shows segregation within the flowing layer. These results shows that larger particles accumulate near the free surface flowing over small particles. During flow small particles easily percolate through the gaps between large particles. The total area fraction in the deep bed is between 0.8 to 0.9 which is close to hexagonal close packed value of 0.9068 . For mixed region the value 

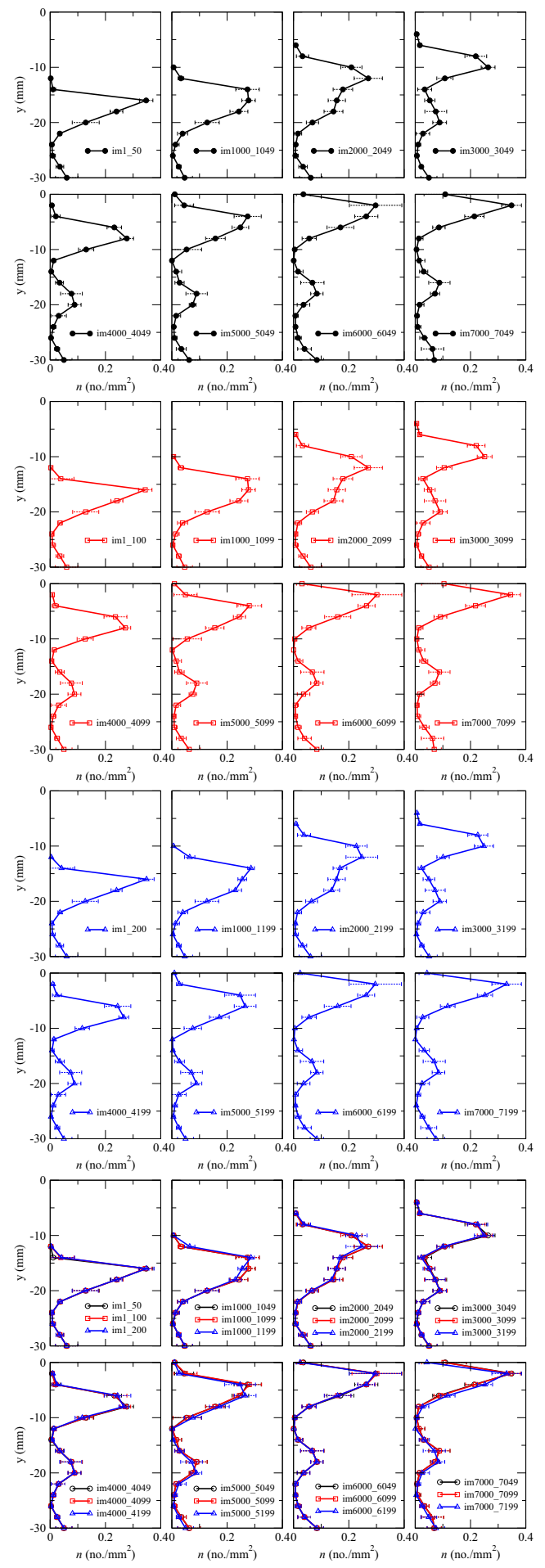

Figure 5. Variation of number density (n) profile within the flowing layer for big particles with time for a set of (a) 50 images (b) 100 images and (c) 200 images (d) 50, $100 \& 200$ images. Equal $\%$ of small $(1 \mathrm{~mm})$ and big $(2 \mathrm{~mm})$ particles. Volume of pouring $200 \mathrm{~g}$. of the area fraction is higher than the hexagonal closed packing. In Hexagonal closed packing layers of particles (sphere) are closely packed so that particles (sphere) in alternating layers overlie one another. Packing fraction (efficiency) is defined as how much space being occupied by the particles present in a unit cell. The area fraction decreases near the free surface for both types of particles. The velocity profiles are linear over most of the layer depth with an exponential decay near the base of the flowing layer [16]. Velocity increases with time and decreases towards the end.

\section{Acknowledgements}

This work was done at IIT Bombay and Project was sponsored by TataSteel Jamshedpur, India.

\section{References}

[1] Makse, H. A., R. C. Ball, H. E. Stanley, and S. Warr, Phys. Rev. E 58, 3 (1988)

[2] Changxing, L., Z. Zhou, R. Zou, D. Pinson, and A. Yu, AIP Conf. Proc. 1542, 767-770 (2013).

[3] Sandip H. Gharat and D. V. Khakhar, AIP Conf. Proc. 1542, 755-758 (2013).

[4] Cizeau, P., H. A. Makse, and H. E. Stanely, Phys. Rev. E, 59, 4408 (1999).

[5] Dolgunin, A. N. Kudy, and A. A. Ukolov, Powder Tech., 96, 211-218 (1998).

[6] Gray, J. M. N. T. and K. Hutter, Contin. Mech. Thermodyn., 9, 341-345 (1997)

[7] Drahun, J. A. and J. Bridgwater, Powder Technol., 36, 39 (1983).

[8] Fan, Y., Y. Boukerkour, T. Blanc, P. B. Umbanhowar, J. M. Ottino, and R. M. Lueptow, Phys. Rev. E, 86, 051305 2012).

[9] Gray, J. M. N. T. and A. R. Thornton, Proc. R. Soc. A, 461, 1447-1473 (2005).

[10] Hajra, S. K. and D. V. Khakhar, Granul. Matter, 13, 475-486 (2011).

[11] Jenkins, J. T. and D. K. Yoon, Phys. Rev. L, 88, 19 (2002).

[12] Khakhar, D. V., J. J. McCarthy, and J. M. Ottino, Phys. Fluids, 9, 3600-3614 (1997).

[13] Khakhar, D. V., J. J. McCarthy, and J. M. Ottino, CHAOS, 9, 3 (1999).

[14] Williams, S. C., Powder Technol., 2, 1320 (1968)

[15] GRAY, J. M. N. T. and C. ANCEY, J. Fluid Mech. 629, 387-423 (2009)

[16] GDR MiDi, Eur. Phys. J. E 14, 341-365 (2004) 